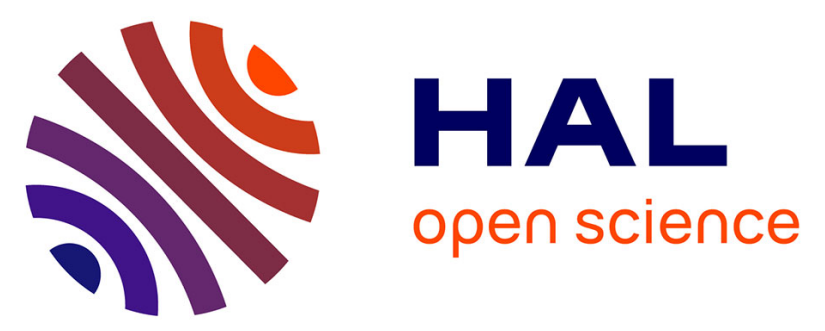

\title{
Tailored stimuli-responsive interaction between particles adjusted by straightforward adsorption of mixed layers of Poly(lysine)-g-PEG and Poly(lysine)-g-PNIPAM on anionic beads
}

Jeremy Malinge, Fanny Mousseau, Drazen Zanchi, Geoffrey Brun, Christophe Tribet, Emmanuelle Marie

\section{To cite this version:}

Jeremy Malinge, Fanny Mousseau, Drazen Zanchi, Geoffrey Brun, Christophe Tribet, et al.. Tailored stimuli-responsive interaction between particles adjusted by straightforward adsorption of mixed layers of Poly(lysine)-g-PEG and Poly(lysine)-g-PNIPAM on anionic beads. Journal of Colloid and Interface Science, 2016, 461, pp.50-55. 10.1016/j.jcis.2015.09.016 . hal-01217105

\section{HAL Id: hal-01217105 \\ https://hal.sorbonne-universite.fr/hal-01217105}

Submitted on 20 Oct 2015

HAL is a multi-disciplinary open access archive for the deposit and dissemination of scientific research documents, whether they are published or not. The documents may come from teaching and research institutions in France or abroad, or from public or private research centers.
L'archive ouverte pluridisciplinaire HAL, est destinée au dépôt et à la diffusion de documents scientifiques de niveau recherche, publiés ou non, émanant des établissements d'enseignement et de recherche français ou étrangers, des laboratoires publics ou privés. 


\title{
Tailored stimuli-responsive interaction between particles adjusted by straightforward adsorption of mixed layers of Poly(lysine)-g-PEG and Poly(lysine)-g-PNIPAM on anionic beads.
}

\author{
Jeremy Malinge ${ }^{1}$, Fanny Mousseau ${ }^{1}$, Drazen Zanchi ${ }^{1,2}$, Geoffrey Brun $^{1}$, \\ Christophe Tribet ${ }^{1}$,Emmanuelle Marie ${ }^{1}$ * \\ ${ }^{1}$ Ecole Normale Supérieure-PSL Research University, Département de Chimie, 24, rue Lhomond, \\ 75005 Paris, France. Sorbonne Universités, UPMC Univ. Paris 06, PASTEUR, F-75005, Paris, \\ France.CNRS, UMR 8640 PASTEUR, F-75005, Paris, France \\ ${ }^{2}$ Université de Paris 7 Denis Diderot, 5 rue Thomas Mann, 75013 Paris, France \\ * Email : emmanuelle.marie@ens.fr; phone : (+33)144322408
}

Abstract : We report a simple and versatile method to functionalize anionic colloid particles and control particle solubility. Poly(lysine)-based copolymers (PLL) grafted with polyethylene oxide (PLLg-PEG) or poly(N-isopropylacrylamide) (PLL-g-PNIPAM) spontaneously adsorb on bare beads dispersed in aqueous solutions of the copolymers. The final composition of the mixed ad-layers formed (i.e. PEG/PNIPAM ratio) was adjusted by the polymer concentrations in solutions. While the (PLL-g-PEG)-coated particles were stable in a wide range of temperature, the presence of PLL-gPNIPAM in the outer layer provided a reversible temperature-triggered aggregation at $32 \pm 1^{\circ} \mathrm{C}$. In the range of PNIPAM fraction going from $100 \%$ (beads fully covered by PLL-g-PNIPAM) down to a threshold $20 \%$ weight ratio (with 80\% PLL-g-PEG), the particles aggregated rapidly to form micrometer size clusters. Below $20 \%$ weight fraction of PLL-g-PNIPAM, the kinetic was drastically lowered. Using PLL derivatives provides a straightforward route allowing to control the fraction of a functional chain (here PNIPAM) deposited on PEGylated particles, and in turn to adjust surface interaction and here the rate of particle-particle aggregation as a function of the density of functional chains. This approach can be generalized to many anionic surfaces onto which PLL is known to adhere tightly, such as glass or silica.

Keywords : Thermoresponsive particle, PLL-g-PEG, PLL-g-PNIPAM 


\section{Introduction}

Polymer coating of particles has become an essential mean to control colloidal stability and particleparticle interactions.[1] When stimuli-responsive chains cover the particles, stable, well-dispersed systems can be switched to aggregation-prone ones. For instance, particles coated with Poly $(\mathrm{N}-$ isopropylacrylamide) undergo temperature-triggered transition above a threshold temperature (cloud point $\sim 32{ }^{\circ} \mathrm{C}$ ).[1-4] Using stimuli-sensitive particles in conditions of time-modulated attractive interaction (pulsed external field) is emerging in material design.[5, 6] The basic perspective is to use the dissipative nature of the system to avoid non-desirable metastable structures [7] or generate new dynamical stationary states which cannot exist without external pulsed drive.[5] Recently, mixed (binary) polymer layers, in which chains of two distinct chemical natures are immobilized on the same surface, were introduced [8-12] as a promising strategy to achieve better control on the reversibility of stimuli-triggered aggregation (upon $\mathrm{pH}$ [13-15], solvent [16, 17] or temperature variation [18]), or to prepare novel objects such as amphiphilic particles [18, 19], and bistable solvent-triggered switch between hydrophobic and hydrophilic states.[16, 20]

Together with the excitement generated by the rich matrix of adjustment parameters of stimuliresponsive and binary polymer layers come the demand for robust experimental strategies enabling their facile preparations on coated particles, with various nature of polymer strands. We propose here the use of comb-like poly(Lysine) derivative as a versatile platform to deposit functional strands on anionic particles. Polymer coated particles are generally prepared by either adsorption of chains (possibly coupled with post-deposition covalent binding, usually called "grafting to"), or by "grafting from" approaches.[8] In the "grafting from" route, polymer chains are grown from initiatorfunctionalized particles. Although grafting-from enables to reach higher polymer densities, this route is more demanding in term of synthesis of mixed binary layers. It requires the sequential polymerizations of two chain types, that shall not interfere.[20-26] The adsorption-based route appears more straightforward to form binary adlayers. Triblock or diblock copolymers were for instance designed to bear in the middle of the chain, one group or a short polymer block that binds to surfaces enabling simultaneous surface attachment of two different strands at 1:1 ratio (i.e. the two blocks flanking the middle moieties).[27, 28] Alternatively, mixed adlayers were obtained from solutions containing two different diblock copolymers having one of their block prone to bind onto surfaces.[29, 30] In water, one versatile strategy to deposit dense hydrophilic polymer layers on a vast variety of negatively charged surfaces is based on the spontaneous adsorption of Poly(Lysine)graft-Poly(ethylene glycol) comb-like copolymer (PLL-g-PEG).[31-34] The polycationic PLL backbone ensures tight Coulomb attachment onto anionic surfaces (glass, plastics, silicium),[31-34] while PEG 
side chains endow surfaces with robust, bio-inert (protein repellent) coverage. Recently, it was shown that azide-containing PLL-g-PEG can be mixed with non-reactive PLL-g-PEG to control the surface density of azido groups for facile "click" attachment of diverse functional groups .[35] We extended this strategy to functionalize commercially available carboxylated polystyrene microspheres (Polybeads, Polyscience) by adsorption of a mixed binary polymer layer comprising a temperature-responsive chain and an hydrophilic (non-responsive) one (Scheme 1).

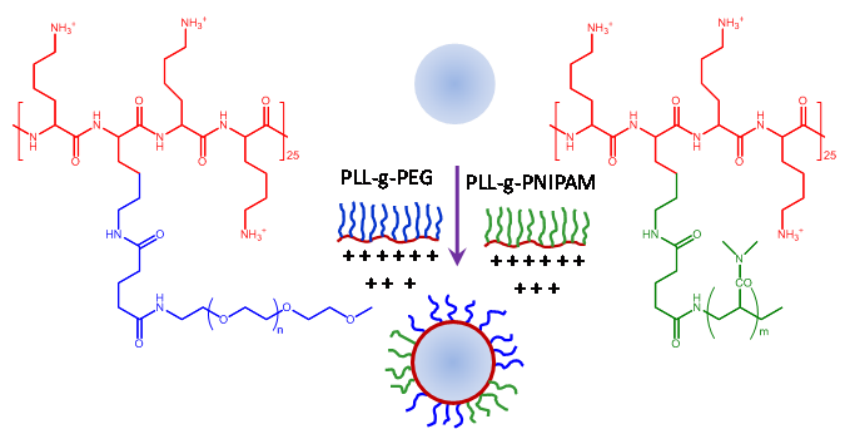

Scheme 1. Schematic representation of the formation of mixed adlayers of PLL-PEG and PLL-PNIPAM on anionic beads.

\section{Materials and methods}

Unless otherwise stated, all chemicals were obtained from commercial sources and used without further purification. Poly(ethylene glycol)s were obtained from Rapp Polymer, Tübingen, Germany. $\mathrm{PLL} \bullet \mathrm{HBr}$ (MW 15-30 kDa) and poly(NIPAM)-NHS were obtained from Sigma Aldrich. Polybead ${ }^{\circledR}$ Carboxylate Microspheres $0.20 \mu \mathrm{m}$ were purchased from Polyscience Inc.

1. Polymer synthesis.

Poly(L-lysine)-graft-poly(ethylene glycol) (PLL-g-PEG). Poly(L-lysine) hydrobromide (19.4 mg, $\mathrm{M}_{\mathrm{w}}=$ 15000 - 30000 g.mol ${ }^{-1}$ ) was reacted with $N$-hydroxysuccinimidyl ester of methoxypoly(ethylene glycol) propionic acid $\left(50 \mathrm{mg}, \mathrm{M}_{\mathrm{w}}=2000 \mathrm{~g} \cdot \mathrm{mol}^{-1}\right)$ in $1 \mathrm{~mL}$ of $50 \mathrm{mM}$ sodium borate buffer solution ( $\mathrm{pH}$ = 8.5). The solution was stirred at room temperature for six hours and dialyzed against water (SlideA-Lyzer, $M_{w}$ cutoff $3500 \mathrm{kDa}$, Thermo Scientific). Lyophilization of the resulting aqueous solution yielded the copolymer as a white solid (59.5 mg, $83 \%$ yield). ${ }^{1} \mathrm{H}$-NMR spectra (Figure S1 in ESI) were recorded at $20^{\circ} \mathrm{C}$ in $\mathrm{D}_{2} \mathrm{O}$ on a Bruker AM 250 SY spectrometer ( $250 \mathrm{MHz}$ ); $\delta$ (ppm): 1.32, 1.53, 1.65 (m, $\left.\mathrm{CH}_{2}, \mathrm{PLL}\right), 2.77$ (s, $\left.\mathrm{CH}_{2}-\mathrm{NH}_{2} \mathrm{PLL}\right), 3.06$ (s, $\left.\mathrm{CH}_{2}-\mathrm{NH}-\mathrm{PEG}\right), 3.29$ (s, -O-CH, $\left.\mathrm{PEG}\right), 3.61$ ( $\mathrm{m}, \mathrm{CH}_{2}, \mathrm{PEG}$ ), 4.19 (s, $\left.\mathrm{CO}-\mathrm{CH}_{2}-\mathrm{NH} \mathrm{PLL}\right)$.

Poly(L-lysine)-graft-poly(N-isopropylacrylamide) (PLL-g-PNIPAM). Poly(L-lysine) hydrobromide (20 $\mathrm{mg}, \mathrm{M}_{\mathrm{w}}=15000-30000 \mathrm{~g} \cdot \mathrm{mol}^{-1}$ ) was reacted with NHS-terminated poly(NIPAM) (104 mg, $\mathrm{M}_{\mathrm{w}}=2000$ 
g.mol $\left.{ }^{-1}\right)$ in $1 \mathrm{~mL}$ of $50 \mathrm{mM}$ sodium borate buffer $(\mathrm{pH}=8.5)$. The solution was stirred for four days at room temperature and was dialyzed against water (Slide-A-Lyzer, $M_{w}$ cutoff $3500 \mathrm{kDa}$, Thermo Scientific). Lyophilization of the resulting aqueous solution yielded the copolymer as a white solid (94.8 mg, 83 \% yield). ${ }^{1} \mathrm{H}$ NMR (Figure S2 in ESI, $250 \mathrm{MHz}, \mathrm{D}_{2} \mathrm{O}$ ), $\delta$ (ppm): 1.05 (s, $\mathrm{CH}_{3} \mathrm{NIPAM}$ ), 1.2-2.2 (m, $\mathrm{CH}_{2}$ PLL), 2.85 (s, NH $\mathrm{NH}_{2}$ PLL), 3.07 (s, NH-CO-NIPAM), 3.81 (s, CH-N-CO NIPAM), 4.20 (s, N-CH-CO PLL).

Rhodamine modified poly(L-lysine)-graft-poly(N-isopropylacrylamide) (Rho-PLL-g-PNIPAM). Poly(Llysine) hydrobromide (40 mg, $M_{w}=15000-30000 \mathrm{~g} \cdot \mathrm{mol}^{-1}$ ) was first reacted with NHS-terminated poly(NIPAM) (208 mg, M $\left.\mathrm{w}_{\mathrm{w}}=2000 \mathrm{~g} \cdot \mathrm{mol}^{-1}\right)$ in $2 \mathrm{~mL}$ of $50 \mathrm{mM}$ sodium borate buffer $(\mathrm{pH}=8.5)$. The solution was stirred for 24 hours. Rhodamine-NHS $\left(10 \mathrm{mg}, \mathrm{M}_{\mathrm{w}}=631 \mathrm{~g} \cdot \mathrm{mol}^{-1}\right)$ diluted in $300 \mu \mathrm{L} \mathrm{DMSO}$ was added to the polymer solution. The reaction medium was then stirred for an additional 24 hours before being dialyzed against water (Slide-A-Lyzer, $M_{w}$ cutoff 3500 kDa, Thermo Scientific). Lyophilization of the resulting aqueous solution yielded the copolymer as a white solid $(201.8 \mathrm{mg}, 81$ $\%$ yield).

\section{UV-visible spectrophotometry.}

UV-vis spectra were recorded on a diode array UV-visible spectrophotometer (Evolution Array, Thermo Scientific) equipped with a Peltier temperature controller $\left( \pm 0.1^{\circ} \mathrm{C}\right)$. To determine the cloud point of each system, the absorbance at $650 \mathrm{~nm}$ was measured as a function of the temperature. Briefly, solutions of PLL-g-NIPAM (2 g. $\mathrm{L}^{-1}$ in millipore water) or functionalized particles ( $20 \mu \mathrm{g} \cdot \mathrm{L}^{-1}$ in 5 $\mathrm{mM}$ phosphate buffer) were subjected to a temperature sweep between $18{ }^{\circ} \mathrm{C}$ and $60{ }^{\circ} \mathrm{C}$ at + $0.25 \mathrm{C} \cdot \mathrm{min}^{-1}$ scan rate (N.B. samples were allowed a 4-minutes equilibration time at each $1^{\circ} \mathrm{C}$ increment). The onset of turbidity above a threshold temperature indicates aggregation. The cloud point was defined as the crossover temperature between the baseline and the tangent to a linear segment of the absorbance vs. T curve just above the onset of the turbidity increase.

\section{Fluorescence spectroscopy.}

Fluorescence spectra were recorded in a Photon Technology International QuantaMaster QM-1 spectrofluorimeter (PTI, Mon-mouth Junction, NJ) that was equipped with a Peltier cell-holder (TLC50, Quantum Northwest, Shoreline, WA). Concentration of beads was adjusted to reach an absorbance of 0.15 at the maximum excitation wavelength (see main text).

4. Dynamic Light Scattering (DLS).

Dynamic light scattering experiments were performed with a Brookhaven BI-200SM apparatus equipped with a multiple tau digital correlator and a $30 \mathrm{~mW}$ diode laser operating at $637 \mathrm{~nm}$. The 
scattered light intensity was measured at $90^{\circ}$ scattering angle and increasing temperatures. Temperature control within $0.1{ }^{\circ} \mathrm{C}$ was achieved using a thermostated bath circulator connected to a temperature probe plunged in the decaline bath nearby the sample cell.

\section{Zetametry.}

Zeta potential were measured in a Zetasizer (Nano ZS, Malvern Instruments Ltd) equipped with a He$\mathrm{Ne}$ laser and temperature controller $\left(+/-0.1^{\circ} \mathrm{C}\right)$. Unless otherwise noticed, aliquots of aqueous dispersion of beads were diluted 10 fold in $50 \mathrm{mM} \mathrm{NaCl}$ solution just before measurements. For temperature-resolved measurement, five measurements were recorded and their standard deviation is reported.

6. Particle coating for DLS measurements.

Commercial dispersions of carboxylate Microspheres (Polybead ${ }^{\circledR}$, diameter $0.20 \mu \mathrm{m}$ ) were dialyzed against water (Slide-A-Lyzer, $M_{w}$ cutoff $3500 \mathrm{kDa}$, Thermo Scientific) to yield the stock suspension S0. An aliquot of SO was freeze-dried and the dried residue was weighted to determine the concentration of beads in SO. Concentration in the stock solution was then adjusted to $18{\mathrm{~g} . \mathrm{L}^{-1}}^{-1}$ in all stock solutions of uncoated beads used in further experiments. In a typical procedure, we added 13 $\mu \mathrm{L}$ of $\mathrm{S} 0$ stock solution of beads into $60 \mu \mathrm{L}$ of a mixed polymer solution (prepared by mixing aliquots of polymer solutions at 10 g.L L $^{-1}$ in 5 mM phosphate buffer of PLL-g-PNIPAM or PLL-g-PEG, or mixed PLL-g-PNIPAM and PLL-g-PEG). The mixed solution was incubated for $5 \mathrm{~min}$. Subsequently, the mixture was purified by size exclusion chromatography (Sephacryl HR 300 column, 2.5 x $1.5 \mathrm{~cm}$ ) preequilibrated with $5 \mathrm{mM}$ phosphate buffer. The typical retention volume of polymer-coated beads was $1.1 \mathrm{~mL}$, making the beads well separated from excess unbound polymer (retention volume of 2.4 $\mathrm{mL}$ ). The concentration of purified beads was determined from the absorbance at $600 \mathrm{~nm}$.

\section{Results and discussion}

Carboxylated polystyrene microspheres (Polybeads, Polyscience) were used as model of negativelycharged organic particles. As determined by DLS and zetametry, dispersions of Polybeads before coating were stable in aqueous buffers, irrespective of temperature in the experimental window $20^{\circ} \mathrm{C}-50^{\circ} \mathrm{C}$ (Figure S3), and the surface charge of particles was highly negative in a wide range of $\mathrm{pH}$ (Table S1). Two PLL based copolymers were synthesized (characterisation in SI): PLL-g-PNIPAM (PolyL-Lysine, Mw=15000 - 30000 g. $\mathrm{mol}^{-1}$ grafted with Poly(N-isopropylacrylamide) side chains, $\mathrm{Mw}=2000$ g.mol ${ }^{-1}$ ) was the temperature-responsive chain, and PLL-g-PEG (PolyEthyleneglycol side grafts, $\mathrm{Mw}=$ $2000 \mathrm{~g} \cdot \mathrm{mol}^{-1}$ ) was the permanently hydrophilic one. First, we determined the concentration at which the surface of Polybeads was saturated. Diluted dispersions of beads $(0.1 \mathrm{~mL}$ of uncoated Polybeads 
at 1 - 2 mg. L $^{-1}$ ) were incubated with increasing amount of polymer (PLL-g-PEG or PLL-g-PNIPAM, from 0 to $60 \mathrm{nmol} . \mathrm{L}^{-1}$ ). After 5 minutes at room temperature, the beads were diluted ( 10 fold in $\mathrm{NaCl} 50$ $\mathrm{mM}$ ) for zeta-potential measurements (Figure 1). Neutralization of the surface charge of Polybeads occurred with increasing polymer concentration (Figure 1) up to a saturation plateau, which reflected adsorption of the cationic PLL derivatives (either PLL-g-PNIPAM or PLL-g-PEG) (Table S1 in SI).

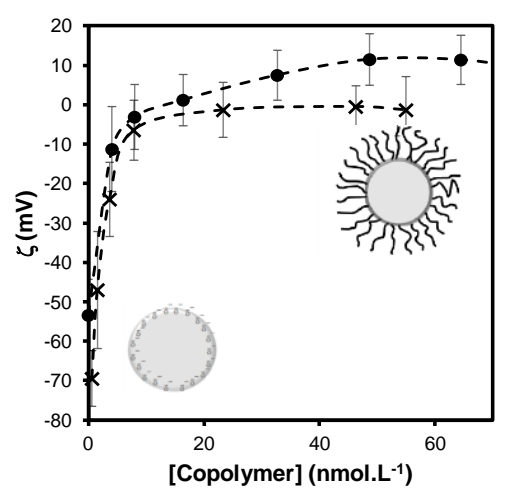

Figure 1. Zeta-potential in $50 \mathrm{mM} \mathrm{NaCl}$ solution of polystyrene Polybeads coated with PLL-g-PNIPAM $(x)$ or PLL-g-PEG $(\bullet)$. Beads (1.2 mg. L $^{-1}$ for PLL-g-PNIPAM or $2.1 \mathrm{mg} . \mathrm{L}^{-1}$ for PLL-g-PEG) were incubated with copolymers concentration as indicated in $\mathrm{x}$-axis.

The near-zero plateau of zeta-potential at high polymer concentrations (slightly positive with PLL-gPEG) indicated neutralization, or even charge reversal by adsorption of PLL-g-PEG. Near complete shielding of the surface charge was reached at a remarkably low concentrations of the copolymers ( $8 \mathrm{nmol} . \mathrm{L}^{-1}$ in both cases) suggesting a high affinity of the PLL derivatives for beads. Using bead diameter and density of $200 \mathrm{~nm}$ and 1.05 respectively, we calculated that the onset of the saturation plateau corresponds to a polymer/surface ratio $>20 \mathrm{mg} \cdot \mathrm{m}^{-2}$. As determined by Santore et al,[33, 34] PLL-g-PEG chains, of similar chain length and similar PEG grafting rate as the present ones, adsorb on bare silica surfaces up to a saturation density of $1.1 \mathrm{mg} \cdot \mathrm{m}^{-2}$. An excess of unbound polymer chains were thus likely present in our preparations. Larger amounts of coated beads were prepared in concentrated solutions ( $18 \mathrm{~g} / \mathrm{L}$ of beads) added into a concentrated polymer solution to reach a copolymer:bead weight ratio arbitrarily fixed at $0.4 \mathrm{~g} / \mathrm{g}, 4.5$-fold above the saturation in Fig. 1 , see methods in ESI). The excess copolymer was removed by size exclusion chromatography (SEC), yielding bead concentrations of $1.8-2$ g. $\mathrm{L}^{-1}$. Zeta-potential was typically of - $5 \mathrm{mV}$ for PLL-g-PNIPAMcoated Polybeads, and $+10 \mathrm{mV}$ for PLL-g-PEG-coated ones, confirming surface saturation.

Temperature-dependant properties of the beads were characterized by UV-vis spectroscopy, dynamic light scattering (DLS), and zetametry. We describe first the dispersion status of beads coated with PLL-g-PNIPAM only (shortnamed BNIPA-100, and diluted to $20 \mu \mathrm{g} \cdot \mathrm{L}^{-1}$ in $5 \mathrm{mM}$ phosphate buffer). 
Aggregation was qualitatively reflected by the decrease of transmittance at $650 \mathrm{~nm}$, that in Figure $2 \mathrm{~A}$ remains high below $\mathrm{T}=32{ }^{\circ} \mathrm{C}$, and undergoes an abrupt decrease above $32{ }^{\circ} \mathrm{C}$. DLS similarly indicated the preservation of the initial diameter of $200 \mathrm{~nm}$ below $32{ }^{\circ} \mathrm{C}$, and formation of micrometer large aggregates at temperatures higher than $32{ }^{\circ} \mathrm{C}$ (Figure. $2 \mathrm{~A}$ ), contrasting with the stability of bare Polybeads (Figure S3 in ESI). Cycling up and down the temperature, between $23^{\circ} \mathrm{C}$ and $38^{\circ} \mathrm{C}$ (Figure 2B) resulted in concomitant up and down variation of absorbance, suggesting that inter-particle sticky bridges are rapidly broken at low T. Upon cooling for longer times (typically 300s), absorbance relaxed down to the initial baseline indicating that full reversibility of re-dispersion of beads was possible (Figure $2 \mathrm{C}$ ).

A

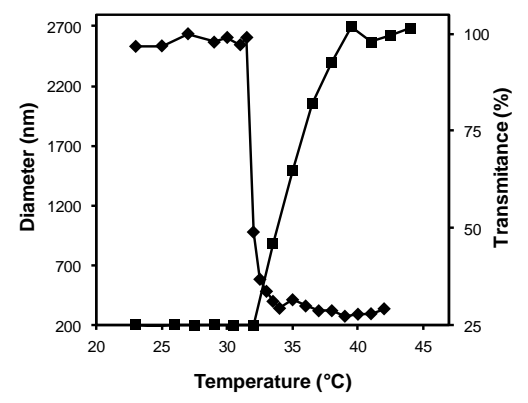

B

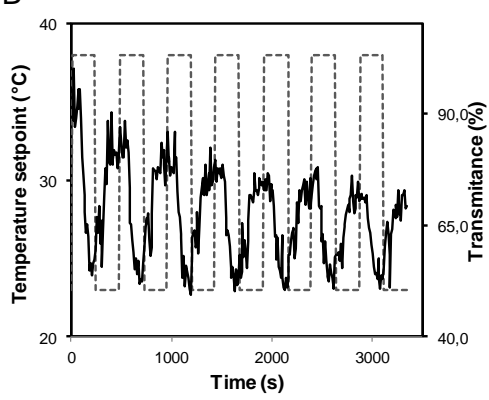

C

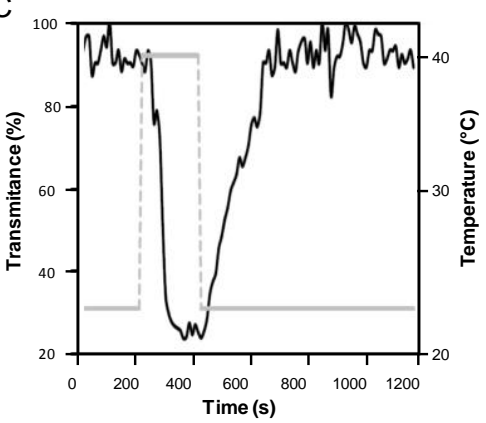

Figure 2. Temperature-triggered aggregation of aqueous dispersions of polystyrene microbeads coated with PLL-g-PNIPAM (20 $\mu \mathrm{g} . \mathrm{L}^{-1}$ in $5 \mathrm{mM}$ phosphate buffer). A) BNIPA-100 characterized by variation of the transmittance at $650 \mathrm{~nm}$ (lozenges) during a temperature sweep $\left(+1{ }^{\circ} \mathrm{C}\right.$ increment every 4-minutes) and variation of apparent hydrodynamic diameter measured by DLS at $90^{\circ}$ angle (squares). B) dispersion of BNIPA-100 characterized by transmittance (650 nm) during heating/cooling cycles. C) resolubilization of BNIPA-50 characterized by transmittance $(650 \mathrm{~nm})$ while subjected to one short (200s) incubation at $40^{\circ} \mathrm{C}$ followed by cooling down to $25^{\circ} \mathrm{C}$.

Zetametry measurements confirmed the temperature-dependent surface properties. At $\mathrm{T}<32{ }^{\circ} \mathrm{C}$, the weakly negative potential $(-5 \mathrm{mV})$ indicated a strong shielding of the charges present at the surface of Polybeads. Zeta-potential dropped to $-25 \mathrm{mV}$ with increasing the temperature above $32{ }^{\circ} \mathrm{C}$ (Figure 3). The marked decrease of zeta-potential at about $32^{\circ} \mathrm{C}$ suggests that shielding by the PNIPAM layer diminishes above $32^{\circ} \mathrm{C}$, which is consistent with transition from good to poor solvent condition. Collapse of the NIPAM chains at high temperature contributes to move the shear plane closer to the surface of PolyBeads, which affect $\zeta$-potential (Figure 3). The negative value of the zeta potential above $32^{\circ} \mathrm{C}$ indicates accordingly that cationic ammonium groups from the adsorbed PLL-gPNIPAM did not compensate the charge coming from carboxylate ions present on the surface of bare Polybeads. Lack of charge neutralization has been reported on systems based on PLL-g-PEG adsorbed 
on silica surfaces[33, 34] and is not surprising. This negative zeta potential is however not sufficient to stabilize the dispersion, which indicates that NIPAM-coated beads are primarily destabilized at high temperature (and aggregate rapidly) due to the increased hydrophobicity of NIPAM with increasing temperature.

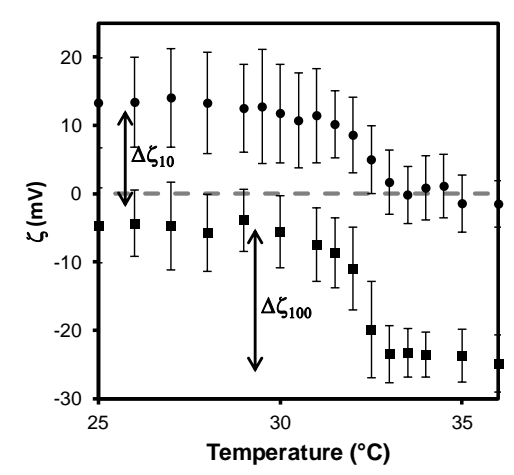

Figure 3. Variation with temperature of $\zeta$-potential of coated Polybeads saturated with PLL-gPNIPAM (BNIPA-100, -), or with a mixture of PLL-g-PEG:PLL-g-PNIPAM (BNIPA-10, •). Beads concentration $=1 \mathrm{mg} \cdot \mathrm{mL}^{-1}$ in $50 \mathrm{mM} \mathrm{NaCl}$.

The versatility of the coating procedure enabled to prepare Polybeads coated by a mixtures of PLL-gPNIPAM and PLL-g-PEG. Beads prepared at varying weight fraction $(f)$ of PLL-g-PNIPAM were shortnamed BNIPA-f. To assess that controlled fraction of PLL-g-PNIPAM can be deposited on the surface of beads, fluorescent derivatives of PLL-g-PNIPAM (rhodamine-modified) were co-adsorbed with PLL-g-PEG, and fluorescent measurements (on SEC purified beads) were performed. After substraction of baseline (fluorescence from uncoated beads), the fluorescence of the rhodaminelabelled PLL-g-PNIPAM in a purified dispersion of coated Polybeads appeared proportional to the fraction $f$ of PLL-g-PNIPAM (Figure 4), indicating that a controlled degree of surface functionalization was achieved.

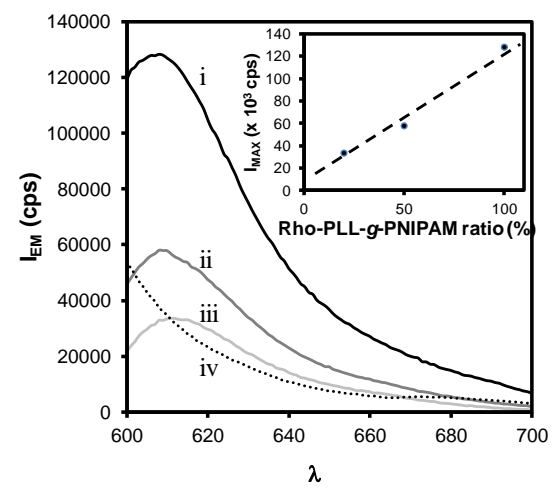


Figure 4. Fluorescence spectra of dispersions of SEC-purified Polybeads coated with mixed PLL-g-PEG: rhodamine-modified PLL-g-PNIPAM added in the coating solution at molar fractions of (i) $100 \%$, (ii) $50 \%$, (iii) $20 \%$ and (iv) $0 \%$. ( $\lambda_{\text {exc }}=550 \mathrm{~nm}$,). Spectra i - iii were obtained by substracting to raw data, the emission of uncoated beads (spectrum iv). Inset: fluorescence intensity $\left(\lambda_{\mathrm{em}}=610 \mathrm{~nm}\right)$ as a function of $\%$ rhodamine-modified PLL-g-PNIPAM.

Temperature-dependant $\zeta$-potential was observed in BNIPA-f, at threshold temperature of $32 \pm 1{ }^{\circ} \mathrm{C}$ (determined at half-drop of zeta-potential, Figure 3 ) irrespective of the fraction $f$ (Table 1 ). This suggest that the presence of PLL-g-PEG on the coated beads did not affect the condition of PNIPAM thermal transition. Beads that were incubated in mixtures containing a lower fraction of PNIPAM displayed higher zeta-potentials and weaker magnitude of zeta-potential variations with T (Table 1). As the PEG moieties are not sensitive to temperature in the experimented window (see Figure S4) this suggests that the fraction of bound PLL-g-PEG increased with decreasing $f$, and contributed to shield the particle's charge.

Table 1. Variation of the zeta-potential with temperature and fraction, $f$, of PLL-g-PNIPAM present in the coating solution.

\begin{tabular}{lccccc}
\hline $\begin{array}{l}\text { PLL-g-PNIPAM } \\
\text { fraction } f(\%)\end{array}$ & 100 & 75 & 50 & 25 & 10 \\
\hline$\Delta \zeta(\mathrm{mV})$ & 20 & 17 & 14 & 14 & 12 \\
$\zeta(\mathrm{mV})\left(\mathrm{T}=20^{\circ} \mathrm{C}\right)$ & -4.3 & 0.5 & 10 & 10 & 12 \\
$\begin{array}{l}\text { Transition } \\
\text { temperature }\left({ }^{\circ} \mathrm{C}\right)\end{array}$ & 32 & 31 & 32 & 30 & 32 \\
\end{tabular}

Surface functionalization with decreasing fraction $f$ of PNIPAM, and increasing fraction of hydrophilic PEG, is expected however to affect the propensity of beads to aggregate at high $T$, which was assessed by DLS. The kinetics of aggregation was thus studied at a temperature somewhat arbitrarily fixed to $40{ }^{\circ} \mathrm{C}$. At time zero, a solution of BNIPA- $f$ ([beads] $=8.5-10.5 \mu \mathrm{g} \cdot \mathrm{L}^{-1}$ in $5 \mathrm{mM}$ phosphate buffer) was plunged in the thermostated cell of the DLS apparatus, and we measured the variation of the hydrodynamic radius as a function of time. Linear increase of radius is representative in Figure $5 \mathrm{~A}$ of the onset of aggregation, that reach micrometer large clustering at longer incubation times (Figure $\mathrm{S} 5$ in ESI). 
A

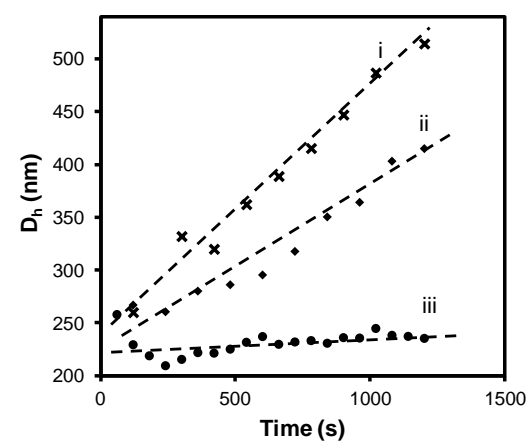

B

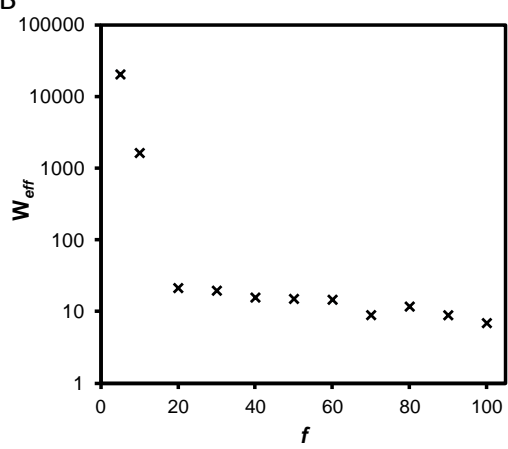

Figure 5. Characteristic features of the aggregation kinetics at $T=40{ }^{\circ} \mathrm{C}$ of BNIPA-f. (A) DLS measurements for BNIPA- $f$ at (i) $f=100 \%$, (ii) $50 \%$, and (iii) $10 \%$ (beads concentration $=8.5-10.5 \mu \mathrm{g} \cdot \mathrm{L}^{-}$ ${ }^{1}$ in $5 \mathrm{mM}$ phosphate buffer); (B) Stability ratio, $W_{\text {eff }}$ plotted as a function of $f$.

At fixed bead concentration, the initial rate of growth of the radius significantly increased with increasing $f$, suggesting that the probability of sticky collision between coated beads increased with increasing fraction of PNIPAM. The rate of variation of radius was translated into the stability ratio, $W$. Slow-aggregation models based on the Smoluchowski theory [36] define $W$ as the ratio between the total number of inter-particle random collisions and the number of collisions leading to aggregation, which at the onset of aggregation (as long as oligomers are the predominant species) is relied to experimental rate by Equation 1.

$D_{h}=D_{1}\left(1+\frac{\alpha \cdot t}{\tau \cdot W}\right) \quad$ where $\quad \tau=\frac{3 . \eta}{4 \cdot k_{B} \cdot T \cdot c_{0}}=\frac{2.10^{11}}{c_{0}}$ (Equation 1$)$

Where $t$ is the time, $D_{1}$ and $D_{h}$ the mean diameters of the dispersed objects measured at time 0 (i.e. isolated beads) and $t$ (beads + aggregates) respectively, $\alpha$ a numerical pre-factor, independent of other physical parameters and $\tau$ the diffusion limited aggregation time that depends on $\eta$ the viscosity of the media, $T$ the temperature, $k_{B}$ the Boltzmann constant and $c_{0}$ the concentration of beads (expressed in $\mathrm{cm}^{-3}$ ). For this study, we arbitrary set $\alpha=1$ to define $W_{\text {eff. }}$ The effective stability ratio $W_{\text {eff }}$ increased gradually with decreasing $f$ from $100 \%$ down to $20 \%$ in the coating mixture. Below $20 \%$ PNIPAM, we observed an abrupt increase of stability ratio (Figure 5, and lack of aggregation in the case of BNIPA-2.5 corresponding to $\left.W_{\text {eff }}>10^{5}\right)$. This control of repulsion/attraction barriers between binary-coated Polybeads indicates that adsorption of mixed PLL-g-PEG:PLL-gPNIPAM provides an effective route to tailor surface functionality and dynamics of aggregation. 


\section{Conclusion}

In the present work we detailed a straightforward coating protocol of negatively charged particles by mixtures of Poly(L-Lysine)-based co-polymers carrying PEG or PNIPAM side chains respectively. With no need for implementation of a specialized chemistry, a system based on PLL derivatives allows direct modulation of the fraction of PNIPAM on particles and enables to control temperature-induced aggregation rates. We believe that this approach can be generalized to most anionic surfaces (PLL derivatives are known to bind readily to glass, plasma-treated polystyrene, or polydimethylsiloxane) as well as to diverse functional side chains (other than PNIPAM), thereby allowing the fine tuning of surface functionalities in mixed polymer layers by a simple "pipetting and incubation" of bare beads in mixed polymer solutions.

\section{Acknowledgements}

This work was mainly supported by ANR DAPPlePur 13-BS08-0001-01, and program Investissement d'Avenir "Dynamo" ANR-11-LABX-0011-01. Authors benefited from access to zeta-potential measurement of "Institut Pierre-Gilles de Gennes » (program "Investissements d'Avenir" ANR-10EQPX-34) with the help of J. Fattacioli.

\section{References}

[1] O.J. Cayre, N. Chagneux, S. Biggs, Stimulus responsive core-shell nanoparticles: synthesis and applications of polymer based aqueous systems, Soft Matter, 7 (2011) 2211-2234.

[2] M.-Q. Chen, T. Serizawa, M. Li, C. Wu, M. Akashi, Thermosensitive Behavior of Poly(Nisopropylacrylamide) Grafted Polystyrene Nanoparticles, Polymer Journal, 35 (2003) 901-910.

[3] M.-Q. Zhu, L.-Q. Wang, G.J. Exarhos, A.D.Q. Li, Thermosensitive Gold Nanoparticles, Journal of the American Society, 126 (2004) 2656-2657.

[4] Q. Zhao, N. Chen, D. Zhao, X. Lu, Thermoresponsive Magnetic Nanoparticles for Seawater Desalination, ACS Applied Materials \& Interfaces, 5 (2013) 11453-11461.

[5] B.A. Grzybowski, C.E. Wilmer, J. Kim, K.P. Browne, K.J.M. Bishop, Self-assembly: from crystals to cells, Soft Matter, 5 (2009) 1110-1128.

[6] J.W. Swan, J.L. Bauer, Y. Liu, E.M. Furst, Directed colloidal self-assembly in toggled magnetic fields, Soft Matter, 10 (2014) 1102-1109.

[7] S.R. Risbud, J.W. Swan, Dynamic self-assembly of colloids through periodic variation of interparticle potentials, Soft Matter, 11 (2015) 3232-3240.

[8] B. Zhao, L. Zhu, Mixed Polymer Brush-Grafted Particles: A New Class of Environmentally Responsive Nanostructured Materials, Macromolecules, 42 (2009) 9369-9383. 
[9] M. Motornov, Y. Roiter, I. Tokarev, S. Minko, Stimuli-responsive nanoparticles, nanogels and capsules for integrated multifunctional intelligent systems, Progress in Polymer Science, 35 (2010) 174-211.

[10] L. Chen, H.A. Klok, "Multifaceted" polymer coated gold nanoparticles, Soft Matter, 9 (2013) 10678-10688.

[11] M.G. Moffitt, Self-Assembly of Polymer Brush-Functionalized Inorganic Nanoparticles: From Hairy Balls to Smart Molecular Mimics, J. Phys. Chem. Lett., 4 (2013) 3654-3666.

[12] I. Luzinov, S. Minko, V.V. Tsukruk, Responsive brush layers: from tailored gradients to reversibly assembled nanoparticles, Soft Matter, 4 (2008) 714-725.

[13] V. Tsyalkovsky, R. Burtovyy, V. Klep, R. Lupitskyy, M. Motornov, S. Minko, I. Luzinov, Fluorescent Nanoparticles Stabilized by Poly(ethylene glycol) Containing Shell for pH-Triggered Tunable Aggregation in Aqueous Environment, Langmuir, 26 (2010) 10684-10692.

[14] M. Motornov, J. Zhou, M. Pita, I. Tokarev, V. Gopishetty, E. Katz, S. Minko, An Integrated Multifunctional Nanosystem from Command Nanoparticles and Enzymes, Small, 5 (2009) 817-820.

[15] M. Motornov, R. Sheparovych, R. Lupitskyy, E. MacWilliams, S. Minko, Responsive colloidal systems: Reversible aggregation and fabrication of superhydrophobic surfaces, Journal of Colloid and Interface Science, 310 (2007) 481-488.

[16] M. Motornov, R. Sheparovych, R. Lupitskyy, E. MacWilliams, O. Hoy, I. Luzinov, S. Minko, Stimuliresponsive colloidal systems from mixed brush-coated nanoparticles, Advanced Functional Materials, 17 (2007) 2307-2314.

[17] F.Y. Wang, L. Cheng, T.H. Chen, D.S. Zhu, Q. Wen, S.W. Wang, Facile Preparation of Polymeric Dimers from Amphiphilic Patchy Particles, Macromolecular Rapid Communications, 33 (2012) 933937.

[18] J.M. Horton, C.H. Bao, Z.F. Bai, T.P. Lodge, B. Zhao, Temperature- and pH-Triggered Reversible Transfer of Doubly Responsive Hairy Particles between Water and a Hydrophobic Ionic Liquid, Langmuir, 27 (2011) 13324-13334.

[19] J. Shan, M. Nuopponen, H. Jiang, T. Viitala, E. Kauppinen, K. Kontturi, H. Tenhu, Amphiphilic Gold Nanoparticles Grafted with Poly(N-isopropylacrylamide) and Polystyrene, Macromolecules, 38 (2005) 2918-2926.

[20] L. Ionov, S. Minko, Mixed Polymer Brushes with Locking Switching, ACS Applied Materials \& Interfaces, 4 (2012) 483-489.

[21] B. Zhao, L. Zhu, Nanoscale phase separation in mixed poly(tert-butyl acrylate)/polystyrene brushes on silica nanoparticles under equilibrium melt conditions, Journal of the American Chemical Society, 128 (2006) 4574-4575.

[22] L. Zhu, B. Zhao, Transmission electron microscopy study of solvent-induced phase morphologies of environmentally responsive mixed homopolymer brushes on silica particles, Journal of Physical Chemistry B, 112 (2008) 11529-11536.

[23] W.K. Li, C.H. Bao, R.A.E. Wright, B. Zhao, Synthesis of mixed poly(epsiloncaprolactone)/polystyrene brushes from Y-initiator-functionalized silica particles by surface-initiated ring-opening polymerization and nitroxide-mediated radical polymerization, RSC Advances, 4 (2014) 18772-18781.

[24] X.M. Jiang, B. Zhao, G.J. Zhong, N.X. Jin, J.M. Horton, L. Zhu, R.S. Hafner, T.P. Lodge, Microphase Separation of High Grafting Density Asymmetric Mixed Homopolymer Brushes on Silica Particles, Macromolecules, 43 (2010) 8209-8217.

[25] S.D. Tang, T.Y. Lo, J.M. Horton, C.H. Bao, P. Tang, F. Qiu, R.M. Ho, B. Zhao, L. Zhu, Direct Visualization of Three-Dimensional Morphology in Hierarchically Self-Assembled Mixed Poly(tertbutyl acrylate)/Polystyrene Brush-Grafted Silica Nanoparticles, Macromolecules, 46 (2013) 65756584.

[26] X.M. Jiang, G.J. Zhong, J.M. Horton, N.X. Jin, L. Zhu, B. Zhao, Evolution of Phase Morphology of Mixed Poly(tert-butyl acrylate)/Polystyrene Brushes Grafted on Silica Particles with the Change of Chain Length Disparity, Macromolecules, 43 (2010) 5387-5395. 
[27] Z.L. Wang, J.T. Xu, B.Y. Du, Z.Q. Fan, Preparation and characterization of V-shaped PS-b-PEO brushes anchored on planar gold substrate through the trithiocarbonate junction group, Journal of Colloid and Interface Science, 384 (2012) 29-37.

[28] Y.Z. Wang, D.Q. Fan, J.P. He, Y.L. Yang, Silica nanoparticle covered with mixed polymer brushes as Janus particles at water/oil interface, Colloid and Polymer Science, 289 (2011) 1885-1894.

[29] D.A. Xiong, G.J. Liu, E.J.S. Duncan, Simultaneous Coating of Silica Particles by Two Diblock Copolymers, ACS Applied Materials \& Interfaces, 4 (2012) 2445-2454.

[30] S. Louguet, B. Rousseau, R. Epherre, N. Guidolin, G. Goglio, S. Mornet, E. Duguet, S.

Lecommandoux, C. Schatz, Thermoresponsive polymer brush-functionalized magnetic manganite nanoparticles for remotely triggered drug release, Polymer Chemistry, 3 (2012) 1408-1417.

[31] G.L. Kenausis, J. Voros, D.L. Elbert, N.P. Huang, R. Hofer, L. Ruiz-Taylor, M. Textor, J.A. Hubbell, N.D. Spencer, Poly(L-lysine)-g-poly(ethylene glycol) layers on metal oxide surfaces: Attachment mechanism and effects of polymer architecture on resistance to protein adsorption, Journal of Physical Chemistry B, 104 (2000) 3298-3309.

[32] N.P. Huang, R. Michel, J. Voros, M. Textor, R. Hofer, A. Rossi, D.L. Elbert, J.A. Hubbell, N.D. Spencer, Poly(L-lysine)-g-poly(ethylene glycol) layers on metal oxide surfaces: Surface-analytical characterization and resistance to serum and fibrinogen adsorption, Langmuir, 17 (2001) 489-498. [33] S. Gon, M. Bendersky, J.L. Ross, M.M. Santore, Manipulating Protein Adsorption using a Patchy Protein-Resistant Brush, Langmuir, 26 (2010) 12147-12154.

[34] S. Gon, B. Fang, M.M. Santore, Interaction of Cationic Proteins and Polypeptides with Biocompatible Cationically-Anchored PEG Brushes, Macromolecules, 44 (2011) 8161-8168.

[35] S.F.M.v. Dongen, J. Janvore, S.S.v. Berkel, E. Marie, M. Piel, C. Tribet, Reactive protein-repellent surfaces for the straightforward attachment of small molecules up to whole cells, Chemical Science, 3 (2012) 3000-3006.

[36] F.D. Evans, H. Wennerström, in: Wiley-VCH (Ed.) The Colloidal Domain where Physics, Chemistry, Biology and Technology meet. 2nd ed.1999, pp. 418-419. 


\section{Figure and table captions}

Scheme 1. Schematic representation of the formation of mixed adlayers of PLL-PEG and PLL-PNIPAM on anionic beads.

Table 1. Variation of the zeta-potential with temperature and fraction, $f$, of PLL-g-PNIPAM present in the coating solution.

Figure 1. Zeta-potential in $50 \mathrm{mM} \mathrm{NaCl}$ solution of polystyrene Polybeads coated with PLL-g-PNIPAM $(x)$ or PLL-g-PEG $(\bullet)$. Beads (1.2 mg. $L^{-1}$ for PLL-g-PNIPAM or $2.1 \mathrm{mg} . \mathrm{L}^{-1}$ for PLL-g-PEG) were incubated with copolymers concentration as indicated in $\mathrm{x}$-axis.

Figure 2. Temperature-triggered aggregation of aqueous dispersions of polystyrene microbeads coated with PLL-g-PNIPAM (20 $\mu \mathrm{g} . \mathrm{L}^{-1}$ in $5 \mathrm{mM}$ phosphate buffer). A) BNIPA-100 characterized by variation of the transmittance at $650 \mathrm{~nm}$ (lozenges) during a temperature sweep $\left(+1^{\circ} \mathrm{C}\right.$ increment every 4-minutes) and variation of apparent hydrodynamic diameter measured by DLS at $90^{\circ}$ angle (squares). B) dispersion of BNIPA-100 characterized by transmittance $(650 \mathrm{~nm})$ during heating/cooling cycles. C) resolubilization of BNIPA-50 characterized by transmittance $(650 \mathrm{~nm})$ while subjected to one short (200s) incubation at $40^{\circ} \mathrm{C}$ followed by cooling down to $25^{\circ} \mathrm{C}$.

Figure 3. Variation with temperature of $\zeta$-potential of coated Polybeads saturated with PLL-gPNIPAM (BNIPA-100, -), or with a mixture of PLL-g-PEG:PLL-g-PNIPAM (BNIPA-10, •). Beads concentration $=1 \mathrm{mg} \cdot \mathrm{mL}^{-1}$ in $50 \mathrm{mM} \mathrm{NaCl}$.

Figure 4. Fluorescence spectra of dispersions of SEC-purified Polybeads coated with mixed PLL-g-PEG: rhodamine-modified PLL-g-PNIPAM added in the coating solution at molar fractions of (i) $100 \%$, (ii) $50 \%$, (iii) $20 \%$ and (iv) $0 \%$. ( $\lambda_{\text {exc }}=550 \mathrm{~nm}$,). Spectra i - iii were obtained by substracting to raw data, the emission of uncoated beads (spectrum iv). Inset: fluorescence intensity $\left(\lambda_{\mathrm{em}}=610 \mathrm{~nm}\right)$ as a function of $\%$ rhodamine-modified PLL-g-PNIPAM. 
Figure 5. Characteristic features of the aggregation kinetics at $T=40{ }^{\circ} \mathrm{C}$ of BNIPA-f. (A) DLS measurements for BNIPA- $f$ at (i) $f=100 \%$, (ii) $50 \%$, and (iii) $10 \%$ (beads concentration $=8.5-10.5 \mu \mathrm{g} \cdot \mathrm{L}^{-}$

${ }^{1}$ in $5 \mathrm{mM}$ phosphate buffer); (B) Stability ratio, $W_{\text {eff }}$ plotted as a function of $f$.

\section{SI figure and table captions}

Table S1. Variation of the $\zeta$-potential of uncoated beads with decreasing $\mathrm{pH}$.

Figure S1. NMR spectra of PLL-g-PEG recorded in $\mathrm{D}_{2} \mathrm{O}$.

Figure S2. NMR spectra of PLL-g-PNIPAM recorded in $\mathrm{D}_{2} \mathrm{O}$.

Figure S3. Apparent hydrodynamic radius of uncoated beads measured by dynamic light scattering at increasing temperature (measurements performed in $5 \mathrm{mM}$ phosphate buffer, with a 4 min thermal stabilization period between each measurement).

Figure S4. Zeta-potential of PLL-g-PEG coated beads, during a T-sweep (increase from room temperature up to $40^{\circ} \mathrm{C}$, with a 4 min thermal stabilization period between each measurement). Beads concentration $=1 \mathrm{mg} \cdot \mathrm{mL}^{-1}$ in $50 \mathrm{mM} \mathrm{NaCl}$.

Figure S5. Temperature-triggered aggregation of BNIPA-100 (a), BNIPA-60 (b) et BNIPA-50 (20 $\mu \mathrm{g} \cdot \mathrm{L}^{-1}$ in $5 \mathrm{mM}$ phosphate buffer. 


\section{Graphical abstract}

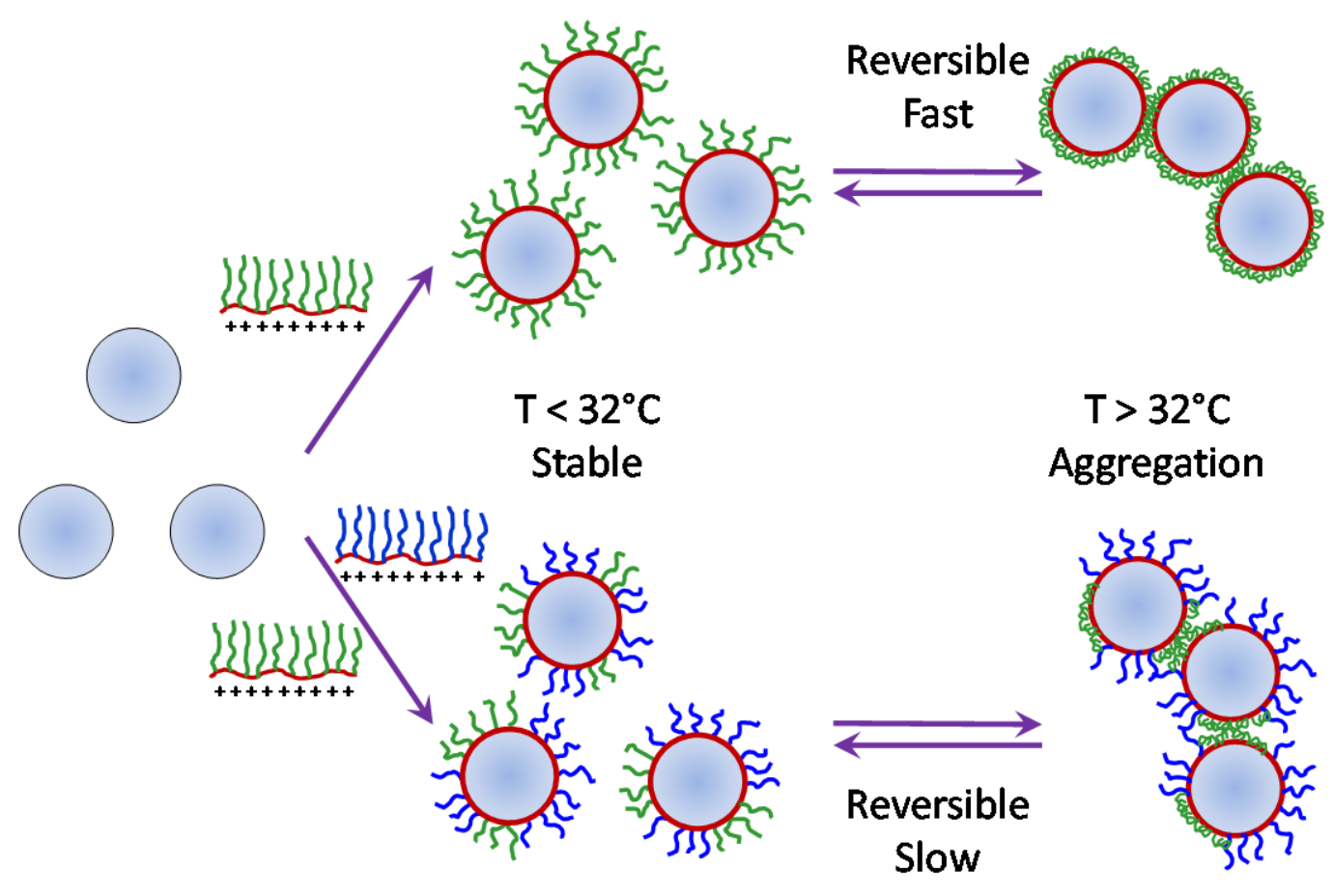




\section{Supporting information}

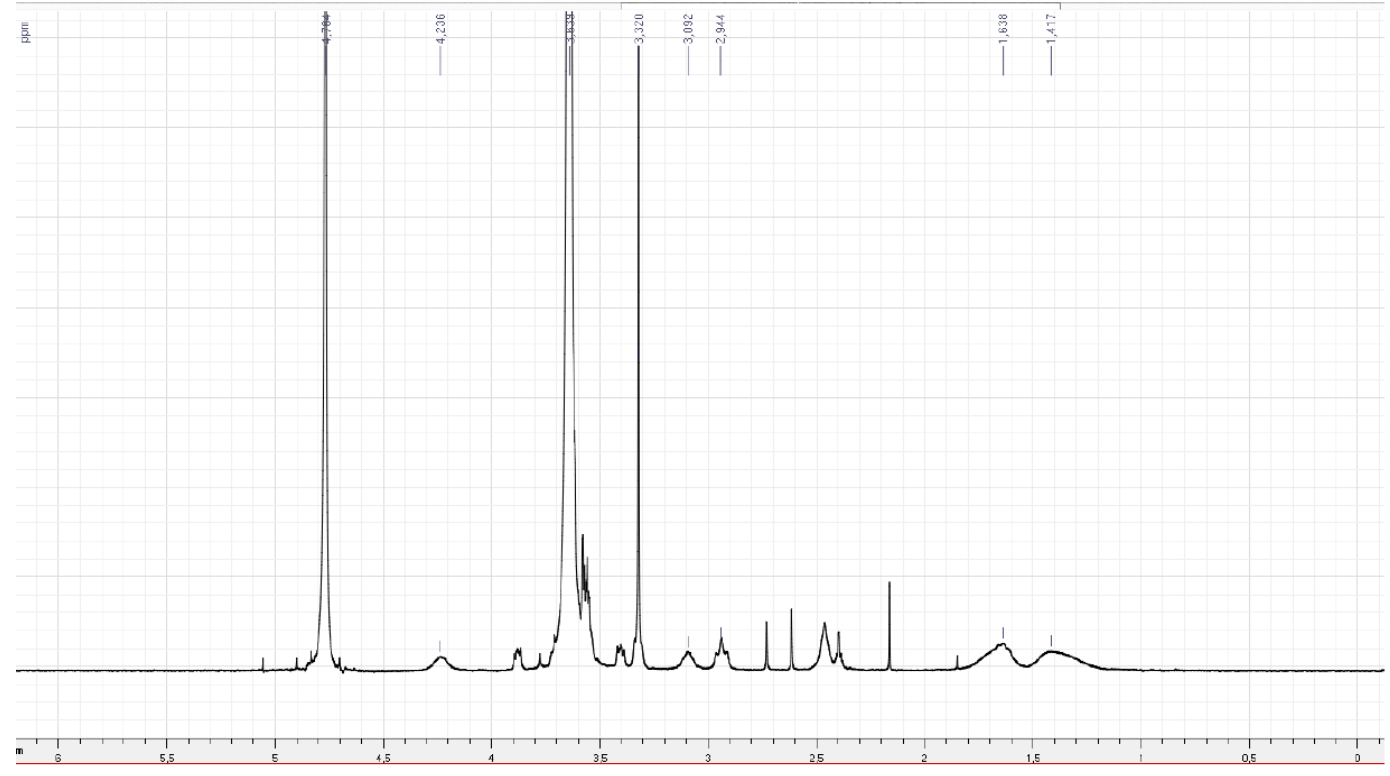

Figure S1. NMR spectra of PLL-g-PEG recorded in $\mathrm{D}_{2} \mathrm{O}$.
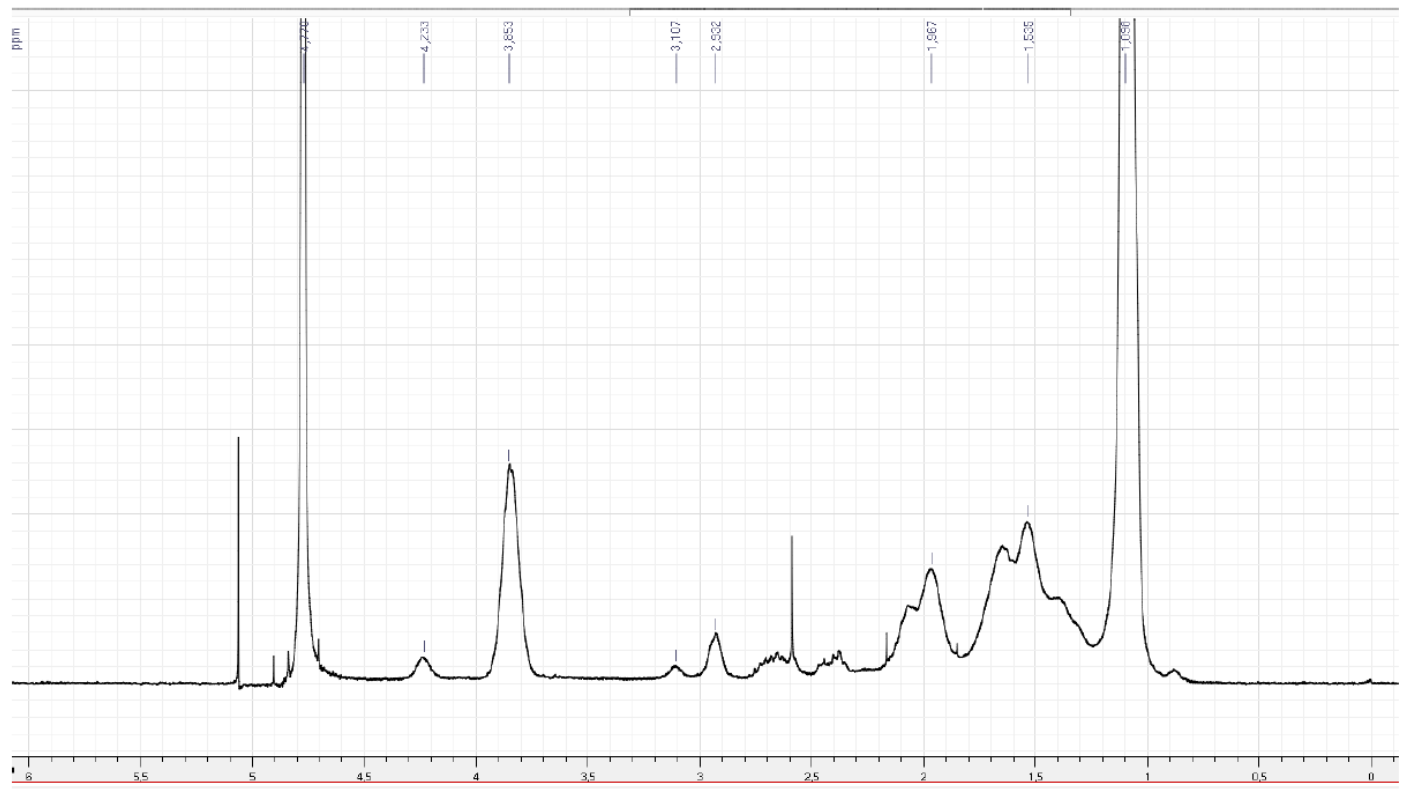

Figure S2. NMR spectra of PLL-g-PNIPAM recorded in $\mathrm{D}_{2} \mathrm{O}$. 
Table S1. Variation of the $\zeta$-potential of uncoated beads with decreasing $\mathrm{pH}$.

$$
\begin{array}{ll}
\hline \mathrm{pH} & \zeta \pm \mathrm{STD}(\mathrm{mV})^{*} \\
\hline 7.5 & -62.6 \pm 17.6 \\
7 & -64.6 \pm 11.9 \\
6.4 & -62.6 \pm 10.6 \\
6 & -54 \pm 10.7 \\
5.5 & -50.1 \pm 11.0 \\
4.7 & -45.3 \pm 7.8
\end{array}
$$

* Measurements performed in phosphate buffer (ionic strength $50 \mathrm{mM}$ )

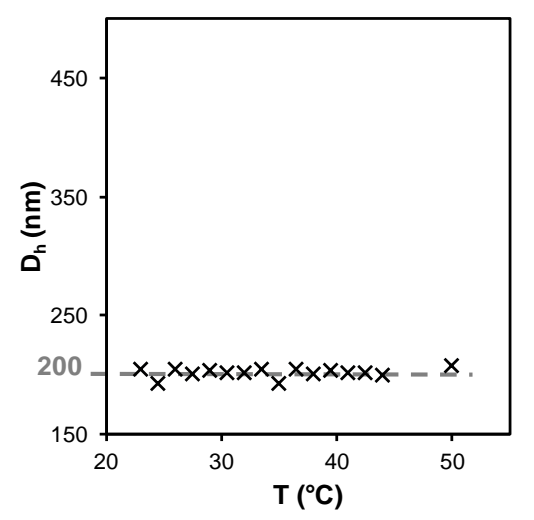

Figure S3. Apparent hydrodynamic radius of uncoated beads measured by dynamic light scattering at increasing temperature (measurements performed in $5 \mathrm{mM}$ phosphate buffer, with a 4 min thermal stabilization period between each measurement). 


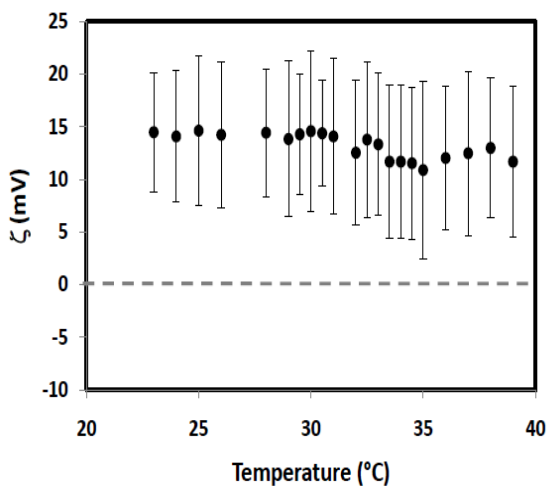

Figure S4. Zeta-potential of PLL-g-PEG coated beads, during a T-sweep (increase from room temperature up to $40^{\circ} \mathrm{C}$, with a 4 min thermal stabilization period between each measurement). Beads concentration $=1 \mathrm{mg} \cdot \mathrm{mL}^{-1}$ in $50 \mathrm{mM} \mathrm{NaCl}$.

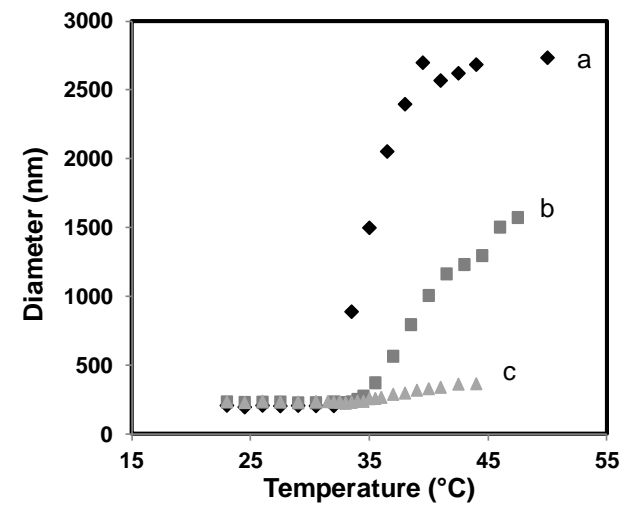

Figure S5. Temperature-triggered aggregation of BNIPA-100 (a), BNIPA-60 (b) et BNIPA-50 (20 $\mu \mathrm{g} \cdot \mathrm{L}^{-1}$ in $5 \mathrm{mM}$ phosphate buffer. 